\title{
Histamine drives severity of innate inflammation via histamine 4 receptor in murine experimental colitis
}

\author{
JB Wechsler ${ }^{1,2}$, A Szabo ${ }^{2}$, CL Hsu $^{2}$, RA Krier-Burris ${ }^{2}$, HA Schroeder ${ }^{2}$, MY Wang $^{1}$, RG Carter $^{2}$, TE Velez $^{2}$, \\ LM Aguiniga ${ }^{3}$, JB Brown ${ }^{1}$, ML Miller ${ }^{2}$, BK Wershil ${ }^{1}$, TA Barrett ${ }^{4}$ and PJ Bryce ${ }^{2}$
}

Ulcerative colitis (UC) patients exhibit elevated histamine, but how histamine exacerbates disease is unclear as targeting histamine 1 receptor $(\mathrm{H} 1 \mathrm{R})$ or $\mathrm{H} 2 \mathrm{R}$ is clinically ineffective. We hypothesized that histamine functioned instead through the other colon-expressed histamine receptor, H4R. In humans, UC patient biopsies exhibited increased H4R RNA and protein expression over control tissue, and immunohistochemistry showed that H4R was in proximity to immunopathogenic myeloperoxidase-positive neutrophils. To characterize this association further, we employed both the oxazolone (Ox)- and dextran sulfate sodium (DSS)-induced experimental colitis mouse models and also found upregulated H4R expression. Mast cell (MC)-derived histamine and H4R drove experimental colitis, as $H_{4 R^{-/}}$mice had lower symptom scores, neutrophil-recruitment mediators (colonic interleukin-6 (IL-6), CXCL1, CXCL2), and mucosal neutrophil infiltration than wild-type (WT) mice, as did MC-deficient $K i t^{W-s h / W-s h}$ mice reconstituted with histidine decarboxylase-deficient $\left(\mathrm{HDC}^{-{ }^{-}}\right.$) bone marrow-derived MCs compared with WT-reconstituted mice; adaptive responses remained intact. Furthermore, $\mathrm{Rag}^{-{ }^{-}-} \times \mathrm{H}_{4} \mathrm{R}^{-1-}$ mice had reduced survival, exacerbated colitis, and increased bacterial translocation than Rag2 ${ }^{-1-}$ mice, revealing an innate protective antibacterial role for H4R. Taken together, colonic MC-derived histamine initiates granulocyte infiltration into the colonic mucosa through H4R, suggesting alternative therapeutic targets beyond adaptive immunity for UC.

\section{INTRODUCTION}

Ulcerative colitis (UC) is a subtype of inflammatory bowel disease (IBD) with chronic symptoms of abdominal pain and bloody diarrhea secondary to colonic inflammation. ${ }^{1}$ The pathogenesis is complex, but a dysfunctional mucosal immune response is critical. ${ }^{2}$ Genome-wide association studies and experimental animal models indicate a role for both innate and adaptive immunity, yet few therapies primarily target innate immunity. ${ }^{2}$ Although these therapeutic advances in treating adaptive immune pathways have revolutionized UC patient outcomes, one in four patients still require colectomy, urging elucidation of other mechanisms that drive inflammation. ${ }^{3,4}$

In healthy colon tissue, inflammatory cells are limited from entering the mucosa until the barrier is breached by luminal insults (e.g., bacteria). ${ }^{5}$ Neutrophils in particular enter the mucosa to kill the bacteria and initiate wound repair; the inflammation ultimately resolves. ${ }^{6}$ A hallmark of active UC is neutrophilic infiltration into the lamina propria that leads to mucosal crypt destruction and neutrophil abscess formation; ${ }^{7}$ neutrophil-derived mediators that cause tissue damage, such as myeloperoxidase (MPO), correlate with disease activity. ${ }^{8,9}$ Although molecules critical to this type of mucosal neutrophil recruitment are well known (e.g., the pleiotropic cytokine interleukin-6 (IL-6) and chemokine CXCL1), ${ }^{10}$ the signals critical to initiating and regulating these innate processes are poorly understood.

The inflammatory cellular milieu of active UC includes a diverse group of granulocytic cells found in $\mathrm{T}$ helper lymphocyte type 2 diseases, including mast cells (MCs),

${ }^{1}$ Department of Pediatrics, Division of Gastroenterology, Hepatology, and Nutrition, Ann \& Robert H. Lurie Children's Hospital of Chicago and Northwestern University Feinberg School of Medicine, Chicago, Illinois, USA. ²Department of Medicine, Division of Allergy and Immunology, Northwestern University Feinberg School of Medicine, Chicago, Illinois USA. ${ }^{3}$ Department of Urology, Department of Microbiology and Immunology, Feinberg School of Medicine, Northwestern University, Chicago, Illinois USA and ${ }^{4}$ Division of Digestive Disease and Nutrition, Department of Medicine, University of Kentucky Health Care, Lexington, Kentucky USA. Correspondence: PJ Bryce (p-bryce@northwestern.edu) 
basophils, and eosinophils., ${ }^{2,11}$ Of these, MCs are uniquely tissue resident and thus poised as early sensors of luminal insults, capable of signaling innate and adaptive immune responses. ${ }^{12,13}$ Activated MCs rapidly release granules containing preformed mediators such as the vasoactive amine, histamine. $^{12,14}$ Although the precise roles of MCs and histamine in UC pathogenesis remain unclear, previous studies implicate both. ${ }^{15}$ For MCs, several reports note correlations between active UC and increased mucosal MC density. ${ }^{16-18}$ Although results from experimental colitis models in MCdeficient animals vary, the overwhelming evidence suggests an important role for MCs and their mediators in driving pathogenesis, as reviewed by Boeckxstaens ${ }^{19}$ in 2015. Elevated mucosal histamine as well as $\mathrm{N}$-methylhistamine metabolite excretion correlate well with patient disease activity. ${ }^{13,20-22}$ Bene et al. ${ }^{23}$ found mice lacking histidine decarboxylase (HDC)--an enzyme necessary for histidine-to-histamine conversion--exhibited reduced inflammation severity to dextran sulfate sodium (DSS)-induced colitis. Additionally, polymorphisms in diamine oxidase, an enzyme critical in histamine breakdown, may affect the outcome of immunemodulator therapeutics in IBD patients. ${ }^{24}$ Though these studies point to a role for MCs and histamine in UC, the precise mechanisms underlying the connections among MCs, histamine, and UC pathogenesis have not yet been fully determined.

Histamine functions through 4 different receptors, histamine 1 receptor $(\mathrm{H} 1 \mathrm{R})-\mathrm{H} 4 \mathrm{R}$, but which receptor(s) is important in UC pathogenesis remains unknown. Small trials utilizing widely available antihistamine blockers for H1R and $\mathrm{H} 2 \mathrm{R}$ in IBD fail to demonstrate clear efficacy, suggesting that they may not be critical in IBD pathogenesis. ${ }^{25}$ Among the remaining histamine receptors, $\mathrm{H} 3 \mathrm{R}$ is not expressed in the gut mucosa, and H4R is expressed in the gut mucosa by mucosal immune cells ${ }^{14}$ (including MCs, eosinophils, T cells, and dendritic cells) as well as nonimmune neuroendocrine cells, enterocytes in the crypt of Lieberkuhn, neurons, and small submucosal blood vessels. Interestingly, prior work suggests that H4R contributes to IBD immunopathogenesis. Varga et al. $^{26}$ found that an H4R antagonist reduced inflammatory responses in 2,4,6-trinitrobenzenesulfonic acid-induced rat colitis. Schirmer et al. ${ }^{27}$ found milder symptoms and histopathologic severity to DSS-induced colitis in $H 4 R^{-1-}$ BALB/c mice compared with wild-type (WT) mice or in WT mice undergoing constant pharmacological H4R blockade; the milder symptoms correlated with a broad reduction in cytokines from restimulated mesenteric lymph node cells and serum IL-6. Although these studies support a potential role for H4R and/or histamine in regulating colitis, the pathways remain unknown, and the key cellular source of histamine remains unidentified.

We demonstrate here that MCs, histamine, and H4R coordinate to promote innate immune-mediated immunopathology and damage associated with mucosal inflammation in colitis. Furthermore, our data show not only that MCs support an innate inflammatory response during colitis distinct from adaptive, $\mathrm{T}$ cell-associated inflammation but also that these two mechanisms are mutually cooperative in maintaining intestinal defenses.

\section{RESULTS}

H4R expression is increased in a subset of untreated patients with active UC and is found in local proximity of activated neutrophils

Histamine is increased in the mucosa of patients with active IBD, but the underlying immune mechanism to drive inflammation via histamine receptors is not well defined. ${ }^{28}$ Previous studies in humans have failed to identify a clear role for either H1R or H2R, ${ }^{28}$ despite both being found on cells in the gut. We hypothesized that H4R--the only other histamine receptor expressed in the gut--may play a critical role. We initially quantified mRNA expression levels of H1R, H2R, and H4R by real-time reverse transcription-PCR in banked clinical samples from newly diagnosed UC patients to determine whether any changes occurred in the histamine receptors compared with non-UC control participants undergoing routine surveillance colonoscopy. Whereas UC patients and control participants had similar $\mathrm{H} 1 \mathrm{R}$ and $\mathrm{H} 2 \mathrm{R}$ mRNA expression in their colon, UC patients exhibited significantly higher H4R expression (Figure 1a); H4R protein levels were also higher by quantifying $\mathrm{H} 4 \mathrm{R}$ immunohistochemical staining (Figure 1b). As a hallmark of UC is neutrophilic infiltration and release of pre-formed MPO-containing granules, we examined MPO protein levels by immunohistochemistry and found significantly higher MPO levels in UC patient colons than control colons (Figure 1c). Comparing serially sectioned immunohistochemical slides from UC patients, we noted areas in which H4R and MPO staining occurred in close proximity to each other (Figure 1d). Taken together, we observe higher H4R expression and increased MPO-positive cells in the colons of newly diagnosed, untreated UC patients compared with control participants, leading to the possibility that histamine, H4R, and granulocytic infiltration may be associated in UC.

\section{H4R drives the severity of experimental colitis}

To explore this potential connection among histamine, H4R, granulocytic infiltration, and severity of colonic inflammation, we utilized two different, well-established experimental models of colitis. Oxazolone (Ox)-induced colitis is a $\mathrm{T}$ cell-driven colitis characterized by a prominent adaptive $\mathrm{T}$ helper cell type 2 (Th2) response with elevated IL-4 and IL-13 as well as a strong innate granulocytic response. DSS-induced colitis, in contrast, has a limited Th1/Th17 response and a profound innate granulocytic response. We first observed that Ox-treated WT mice exhibited significantly higher H4R expression than untreated control WT mice, mirroring our observations in the patient population (Supplementary Figure S1a online). Additionally, isolated RNA from untreated murine colonic epithelial cells ( $>80 \%$ epithelial cell adhesion molecule positive by flow cytometry) did not have detectable H4R expression (Supplementary Figure S1b) despite robust H1R and H2R 

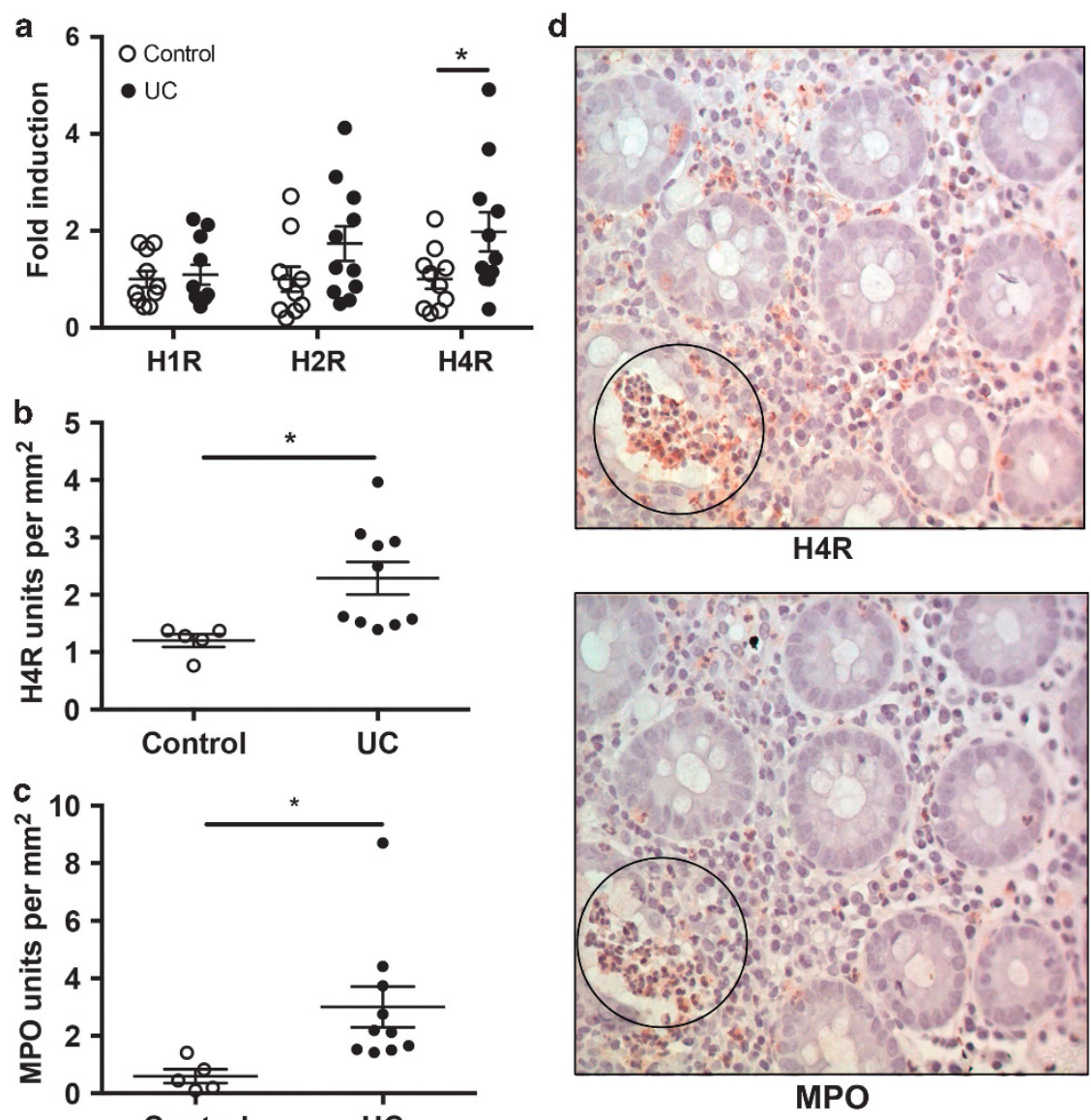

Figure 1 Histamine 4 receptor ( $\mathrm{H} 4 \mathrm{R})$ is increased in active ulcerative colitis (UC) and regionally colocalizes with MPO ${ }^{+}$cells. (a) Quantitative real-time PCR of RNA isolated from biopsy lysates from non-UC individuals (Control) and UC patients displayed as fold induction vs. control for H1R, H2R, and H4R. (b) Relative intensity quantification of H4R immunohistochemistry (IHC) from non-UC individuals and UC patients displayed as relative units per $\mathrm{mm}^{2}$. (c) Relative intensity quantification of myeloperoxidase (MPO IHC for non-UC individuals and UC patients in relative units per mm². (d) Example of H4R/MPO staining on serial sections of colon tissue (circle indicates area of regional colocalization). ${ }^{*} P<0.05$.

expression, suggesting that $\mathrm{H} 4 \mathrm{R}$ tissue expression in the colon is primarily restricted to immune cells, as has previously been described. ${ }^{25}$ To test whether H4R functionally contributed to disease pathogenesis, we examined clinical disease severity between WT and H4R-deficient knockout $\left(H 4 R^{-/-}\right)$mice by measuring both changes in weight and degree of bloody diarrhea (disease activity index score). In both models, $H 4 R^{-/-}$ mice had reduced weight loss over the course of 3 days compared with WT mice (Figure 2a,e) and reduced disease activity index scores at the peak of disease (Figure $\mathbf{2 b}, \mathbf{f}$ ). This protection against clinical severity in $H 4 R^{-\prime-}$ correlated with a decrease in tissue damage, as the colons of $H 4 R^{-/-}$mice had significantly reduced histology scores (Figure $\mathbf{2 c}, \mathbf{g}$ ) compared with WT colons. Notably, among the principal components evaluated for the histology score, $H 4 R^{-1-}$ mice had significantly lower cellular infiltration and decreased overall mucosal damage in both Ox- and DSS-induced colitis (Figure $\mathbf{2 d}, \mathbf{h}-\mathbf{j}$ ). Taken together, H4R may play a role in driving key aspects of the inflammatory responses--in particular, tissue damage-promoting cellular infiltration-observed in both colitis models.

\section{H4R drives neutrophil recruitment to the mucosa}

Neutrophil infiltration is a hallmark of UC; in particular, activated neutrophils attacking mucosal crypts create the characteristic neutrophil abscesses. We therefore quantified neutrophil abscess formation in the tissues of WT and $H 4 R^{-/-}$ and found that $H 4 R^{-1-}$ colons had significantly less frequent neutrophil abscesses in the mucosa (area above the submucosal space) than WT in both Ox- and DSS-induced colitis (Figure 3a and Supplementary Figure S2). These data suggest that H4R plays a role in neutrophil movement and migration into the mucosa, where neutrophils elicit the most tissue damage in colitis. Focusing on the Ox model--the model most linked to Th2-type responses observed in UC--we sought to more fully characterize this neutrophil infiltration. Consistent with neutrophil abscess frequency, $H 4 R^{-1-}$ colons contained fewer MPO-activated mucosal neutrophils compared with WT (Figure 3b,c). One possible explanation is that neutrophil migration signals are affected in the absence of H4R signaling. Indeed, $H 4 R^{-1-}$ colon lysates had significantly reduced levels of the neutrophil-attracting molecules IL-6, CXCL1, and CXCL2 than WT (Figure 3d); in DSS-induced colitis, 

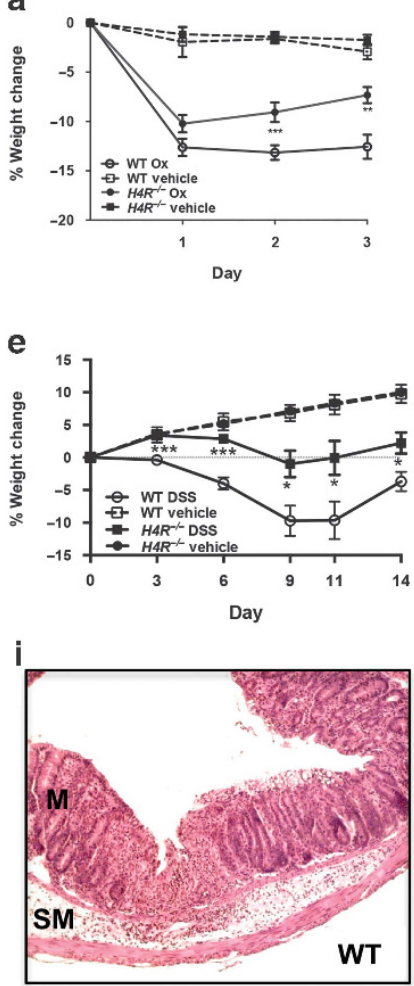

b

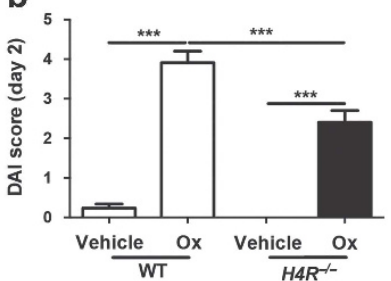

f
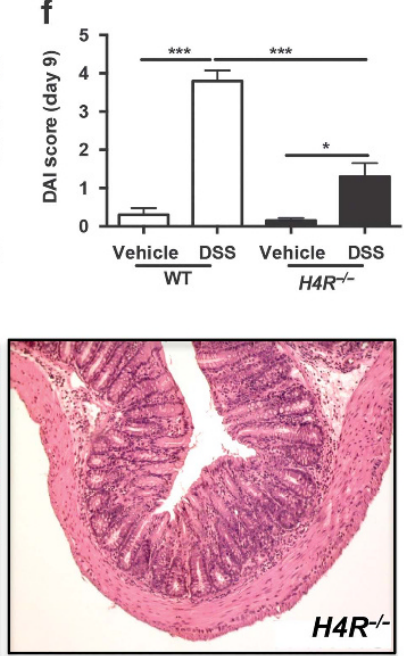
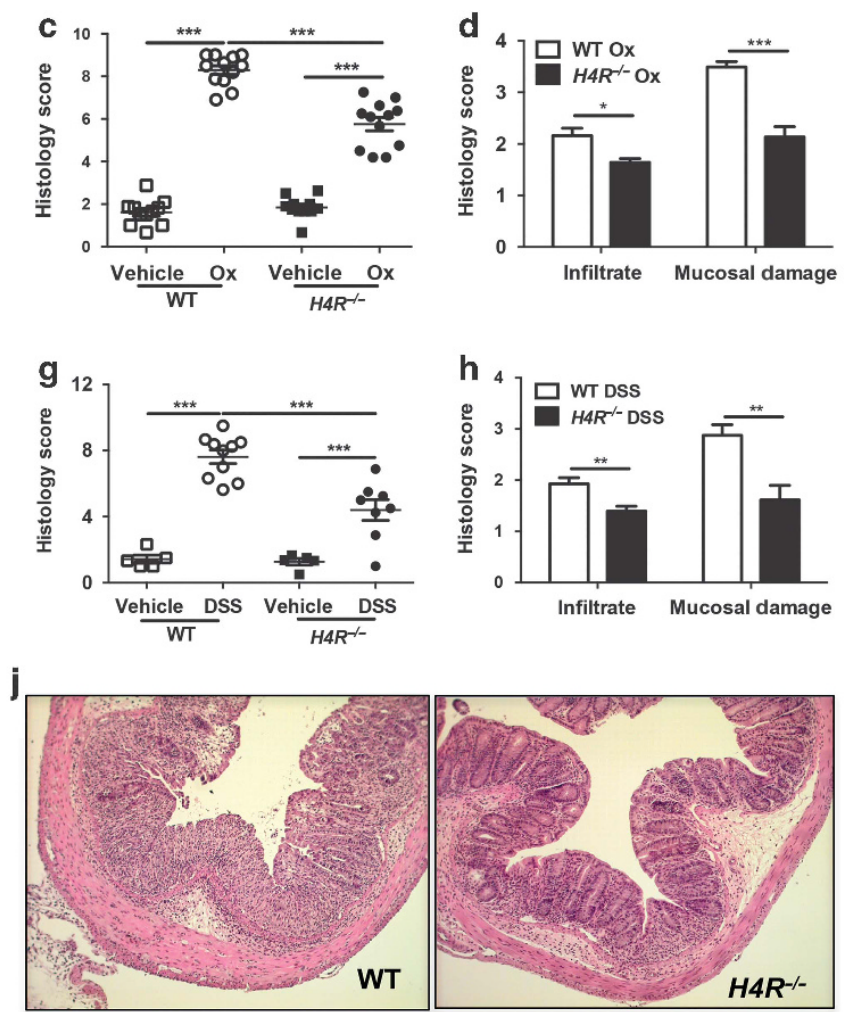

Figure 2 Histamine 4 receptor (H4R) drives clinical and histologic severity in oxazolone (Ox)-and dextran sulfate sodium (DSS)-induced experimental colitis. (a-d, i) Ox-induced and (e-h, j) DSS-induced colitis experiments. (a, e) Percentage weight change in BALB/c vs. H4R ${ }^{-1-}$ mice $(n=16-26$ mice/group). (b, f) Disease activity index (DAI) score for wild-type (WT) and H4R ${ }^{-/}$mice. (c, g) Composite histology score for WT and H4R ${ }^{-/-}$mice. (d, h) Component histology score for cellular infiltrate and mucosal damage in WT and H4R ${ }^{-/-}$mice. (i, j) Representative histology in WT and H4R ${ }^{-/-}$ mice. ${ }^{*} P<0.05,{ }^{* *} P<0.01,{ }^{* *} P<0.001$. Data represented as mean \pm s.e.m. from 3-4 independent experiments.

a

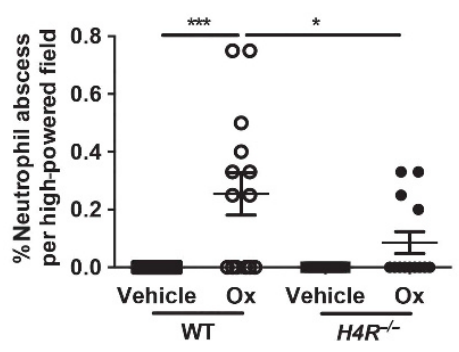

d

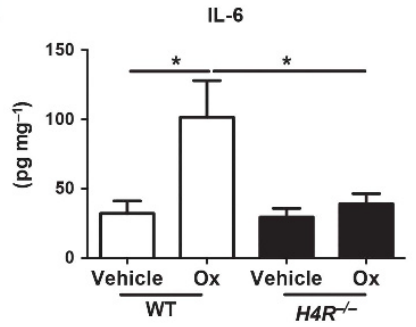

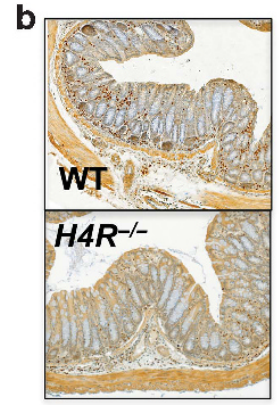

C
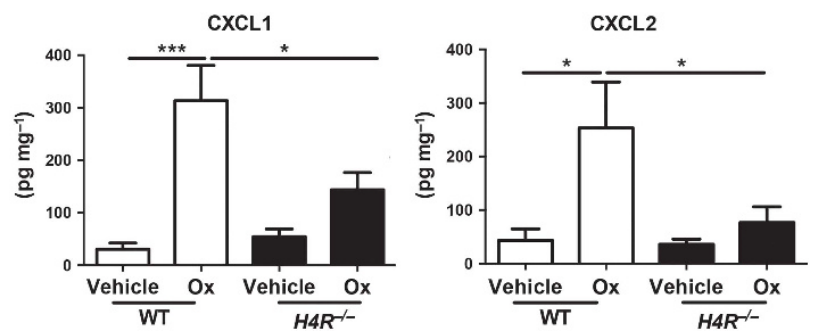

Figure 3 Histamine 4 receptor $(\mathrm{H} 4 \mathrm{R})$ signaling allows for neutrophil recruitment into the mucosa during experimental colitis. (a) Percentage neutrophil abscesses/high-powered field (HPF) present within colon sections (3-5 sections/animal). (b) Representative myeloperoxidase (MPO)

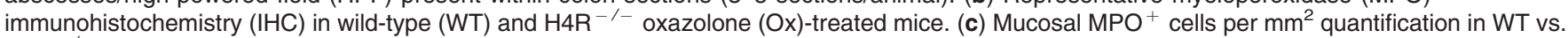
H4R ${ }^{-/-}$Ox-treated mice. (d) Colonic interleukin-6 (IL-6), CXCL1, and CXCL2 protein quantification by ELISA. ${ }^{\star} P<0.05,{ }^{* \star *} P<0.001$. Data represented as mean \pm s.e.m. from three independent experiments. 
$H 4 R^{-1-}$ colon lysates had significantly lower IL-6 and CXCL1 than WT (Supplementary Figure S3). Taken together, these data suggest that H4R plays a role upstream of recruiting neutrophils into colon tissue at least in part by regulating neutrophil-attracting chemokine/cytokine production.

\section{MC-derived histamine drives severity of experimental colitis at least in part by recruiting neutrophils into the colonic mucosa}

To next determine whether histamine, the ligand for H4R, was also required for neutrophil infiltration into the colon after Oxinduced colitis, we examined Ox-induced colitis in histaminedeficient $H D C^{-/-}$and WT mice and found that $H D C^{-1-}$ mice exhibited less severe colitis with significantly reduced weight loss, disease activity index scores, and histology scores (Figure 4a-c), similar to $H 4 R^{-1-}$ mice. Importantly, $H D C^{-/-}$ mice recapitulated the reduced neutrophil infiltration observed in the $H 4 R^{-/-}$mice with significantly lower neutrophil abscess formation and lower IL-6, CXCL1, and CXCL2 expression (Figure 5a,b). To rule out whether this effect of histamine on exacerbating colitis worked through either of the other gut-expressed histamine receptors, we performed the Ox-induced colitis model in H1R/H2R double-knockout mice $\left(H 1 R^{-1-} \times H 2 R^{-/-}\right)$and found similar weight loss, disease activity index scores, and histology scores between $H 1 R^{-1-} \times H 2 R^{-1-}$ and control WT mice (Supplementary Figure S4). Thus, the effect of histamine on proinflammatory clinical and histologic severity in experimental colitis is likely independent of H1R or H2R effects in the mucosa and functions instead through H4R-mediated processes.
MCs and basophils are considered the predominant histamine sources. As MCs reside in tissues whereas basophils mainly circulate in blood, MC-derived histamine may play a role in coordinating neutrophil infiltration and exacerbating colitis. We tested this by reconstituting MC-deficient Sash mice with bone marrow-derived MCs (BMMCs) grown from either $\mathrm{WT}$ or $H D C^{-/-}$mice and then challenging the recipient mice with the Ox-driven model; to control for procedural stress, vehicle-reconstituted mice were first sensitized to $\mathrm{Ox}$ and then challenged with vehicle. Compared with WT BMMC-reconstituted Sash mice, those reconstituted with $H D C^{-/-}$BMMCs exhibited decreased disease severity, neutrophil abscesses, and neutrophil-attracting molecules (Figures $4 \mathbf{d}-\mathbf{f}$ and $\mathbf{5 c}, \mathbf{d})$. Collectively, MC-derived histamine plays a previously unappreciated role in regulating innate immune-mediated inflammation through H4R within the colonic mucosa.

\section{T-cell responses during experimental colitis are} independent of H4R, histamine, or MC-derived histamine The pathogenesis and severity of Ox or DSS experimental colitis is often attributed to adaptive immunity, in particular the T-cell response. ${ }^{29-31}$ To test whether the histamine/H4R axis had any effect on adaptive immunity that could account for exacerbating colitis, we examined several $\mathrm{T}$ cell-associated responses in the colon after Ox- or DSS-induced colitis. Despite significant differences in disease severity between WT and $H 4 R^{-1-}$ mice in the Th2-associated Ox model, they exhibited similar Th2-driven IL-4 and IL-13 expression and CD4 ${ }^{+}$T-cell frequency within the $\mathrm{CD} 45^{+}$pool of lamina propria mononuclear cells at day 3 (Figure 6a,b). Similarly, T cell-driven IL-17A was also similar between $H 4 R^{-1-}$ and WT mice in the
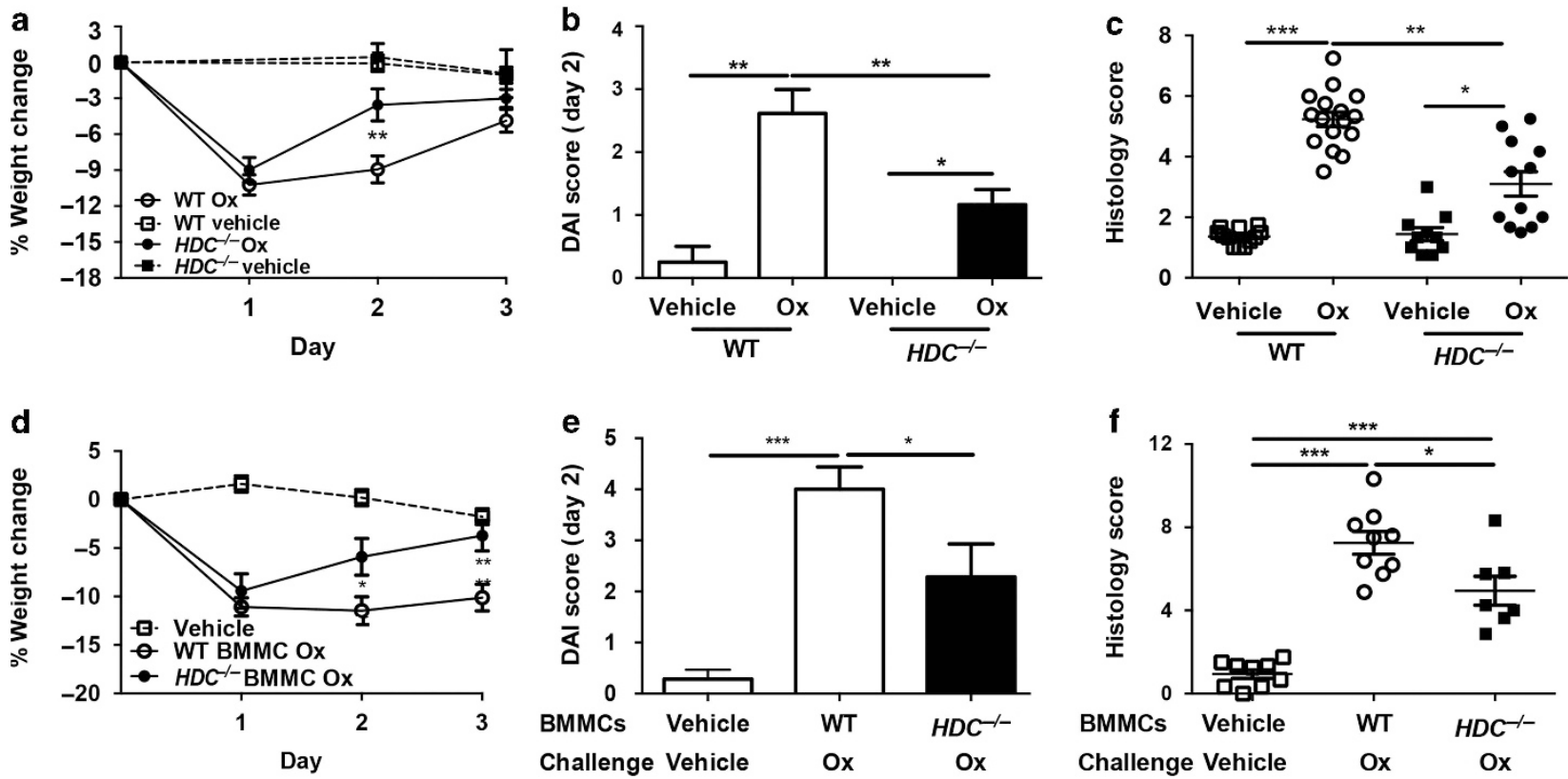

Figure 4 Mast cell (MC)-derived histamine drives clinical and histologic severity of experimental colitis. (a) Percentage weight change in oxazolone (Ox)- or vehicle-treated wild-type (WT) and histidine decarboxylase-deficient $\mathrm{HDC}^{-/-}$) mice $(n=11-24$ mice/group). (b) Disease activity index (DAI) score in Ox- or vehicle-treated WT and $\mathrm{HDC}^{-/-}$mice (day 2). (c) Histology score in Ox- or vehicle-treated WT and HDC ${ }^{-/-}$mice (day 3). (d) Percentage weight change in Ox- or vehicle-treated WT or $\mathrm{HDC}^{-1-}$ bone marrow-derived MC (BMMC)-reconstituted Sash mice $(n=7-10 \mathrm{mice} / \mathrm{group})$. (e) DAI score in Ox- or vehicle-treated WT or HDC ${ }^{-1-}$ BMMC-reconstituted Sash mice (day 2). (f) Histology score in Ox- or vehicle-treated WT or HDC ${ }^{-\prime}$ BMMC-reconstituted Sash mice (day 3). ${ }^{\star} P<0.05,{ }^{\star \star} P<0.01,{ }^{\star \star \star} P<0.001$. Data represented as mean \pm s.e.m. from three independent experiments. 

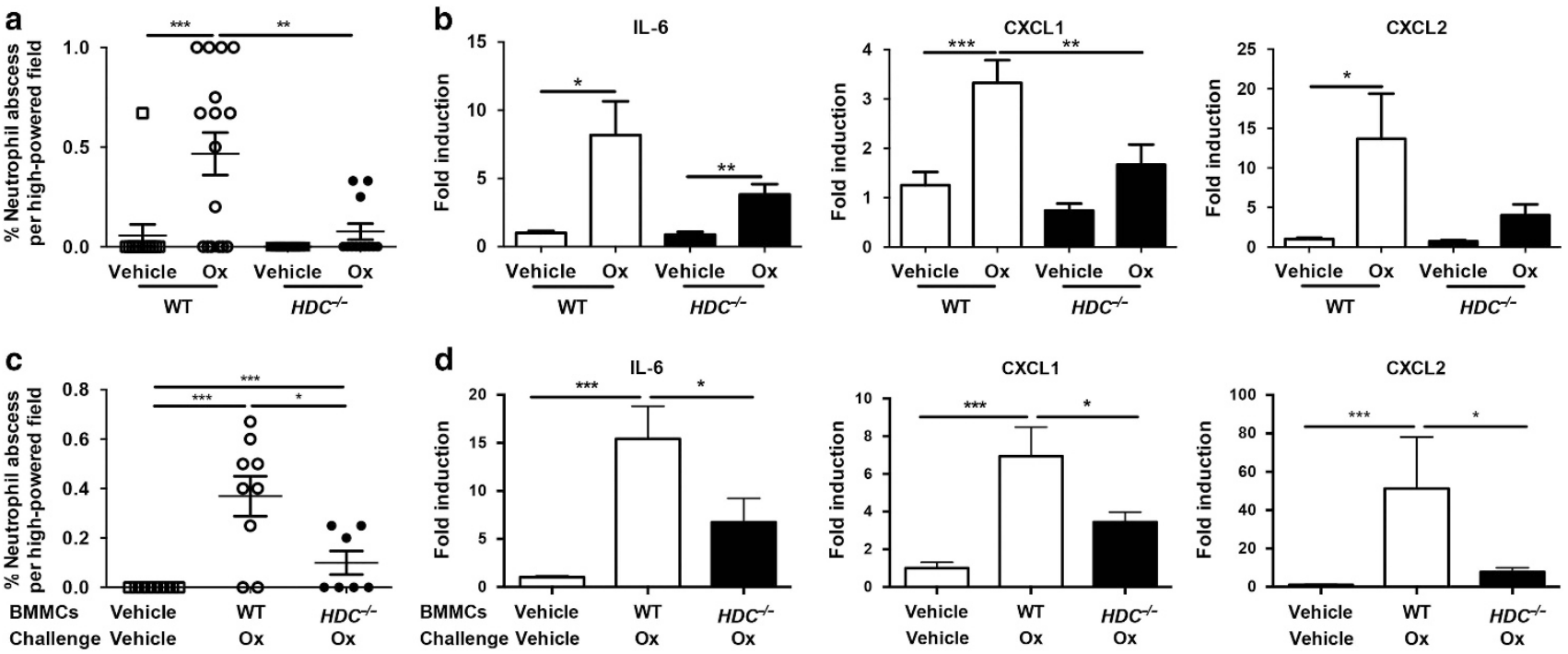

Figure 5 Mast cell (MC)-derived histamine aids in neutrophil recruitment into the mucosa during experimental colitis. (a) Percentage neutrophil abscesses/high-powered field (HPF) present within colon sections ( $n=3-5$ sections/animal) from oxazolone (Ox)- or vehicle-treated wild-type (WT) and histidine decarboxylase-deficient ( $\mathrm{HDC}^{-1-}$ ) mice. (b) Quantitative real-time PCR of colon lysates for interleukin-6 (IL-6), CXCL1, and CXCL2 from Ox- or vehicle-treated WT and $\mathrm{HDC}^{-/-}$mice; fold induction shown relative to vehicle. (c) Percentage neutrophil abscesses/HPF present within colonic sections ( $n=3-5$ sections/animal) from Ox- or vehicle-treated WT and $\mathrm{HDC}^{-1-}$ bone marrow-derived MC (BMMC)-reconstituted Sash mice. (d)

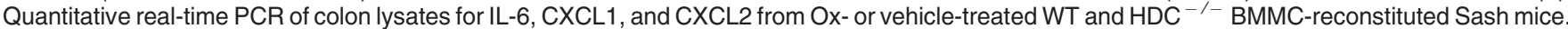
${ }^{\star} P<0.05,{ }^{* *} P<0.01,{ }^{* \star *} P<0.001$. Data represented as mean \pm s.e.m. from 3 independent experiments.
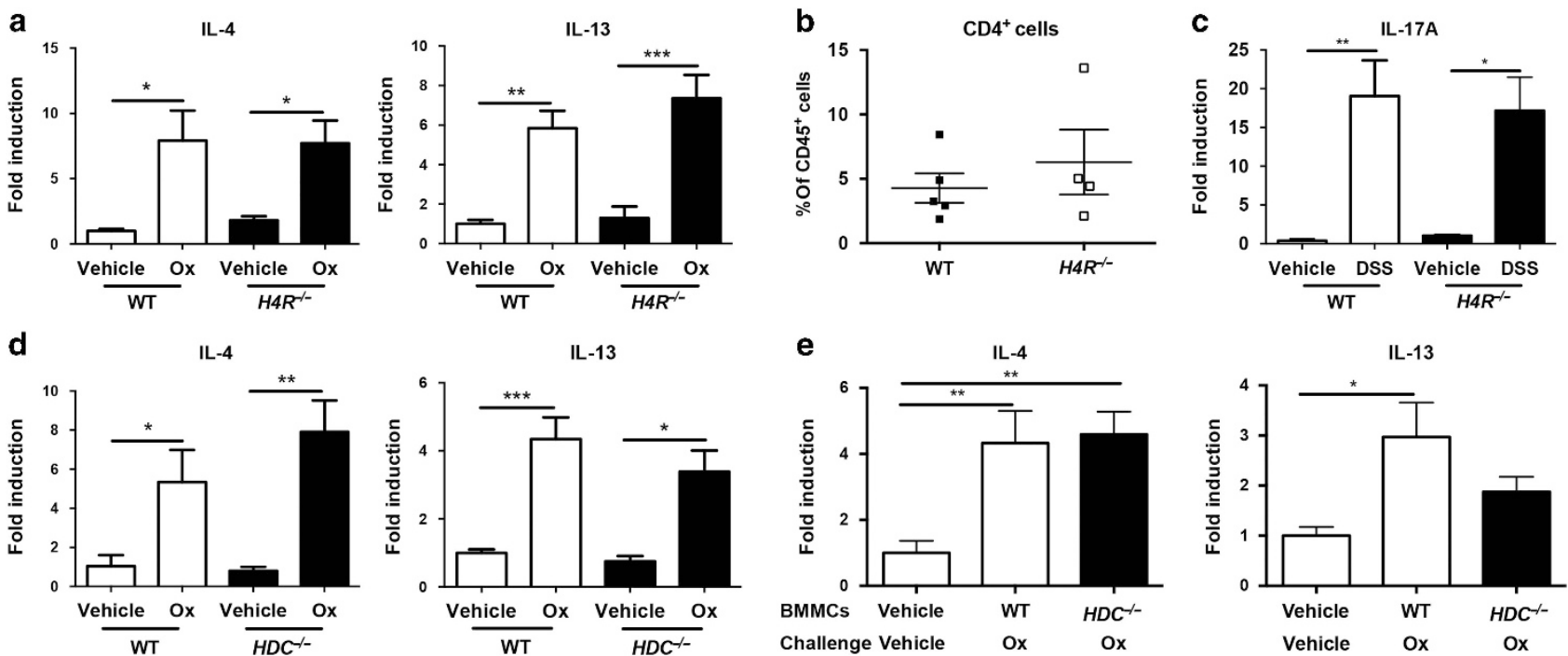

Figure 6 T-cell responses during experimental colitis are unaffected by mast cell (MC)-derived histamine or histamine 4 receptor (H4R). (a) Quantitative real-time PCR for T cell-derived cytokines in colonic lysates from oxazolone (Ox)- or vehicle-treated wild-type (WT) and $\mathrm{H}_{4} \mathrm{R}^{-/-}$mice (day 3$)$. (b) Frequency of $\mathrm{CD} 4{ }^{+} \mathrm{CD} 45^{+}$lamina propria mononuclear cells (LPMCs) isolated from colon from Ox- or vehicle-treated WT and H4R ${ }^{-/-}$mice (day 3 ). (c) Quantitative real-time PCR for interleukin-17A (IL-17A) in colonic lysates from dextran sulfate sodium (DSS)- or vehicle-treated WT and H4R ${ }^{-1-}$ mice (day 9). (d) Quantitative real-time PCR from colonic lysates from Ox- or vehicle-treated WT vs. histidine decarboxylase-deficient (HDC ${ }^{-1-}$ ) mice (day 3 ). (e) Quantitative real-time PCR from colonic lysates from Ox- or vehicle-treated WT or $\mathrm{HDC}^{-/-}$bone marrow-derived MC (BMMC)-reconstituted Sash mice (day 3). ${ }^{*} P<0.05,{ }^{* *} P<0.01$. Data represented as mean \pm s.e.m. from three independent experiments, except $\mathbf{b}$ (two independent experiments).

Th1/Th17-associated DSS-induced colitis at day 9 (Figure 6c). $\mathrm{T}$ cell-dependent contact hypersensitivity responses (ear swelling) to Ox challenge were also similar between sensitized $H 4 R^{-l-}$ and WT mice (Supplementary Figure S5). We also observed no changes in Th2-driven IL-4 and IL-13 expression between $H_{D C}{ }^{-1-}$ and WT mice, or between WT BMMC- and $H D C^{-/-}$BMMC-reconstituted Sash mice (Figure 6d,e). Taken together, the competent $\mathrm{T}$-cell responses observed in the $H 4 R^{-/-}, H D C^{-/-}$, and $H D C^{-/-}$BMMC-reconstituted Sash mice suggest the histamine/H4R axis is not required for adaptive T-cell responses in these colitis models.

\section{H4R plays a key protective antibacterial role in innate immune signaling}

As both adaptive T-cell and innate immune responses likely contribute to inflammation in colitis, we wondered what the 

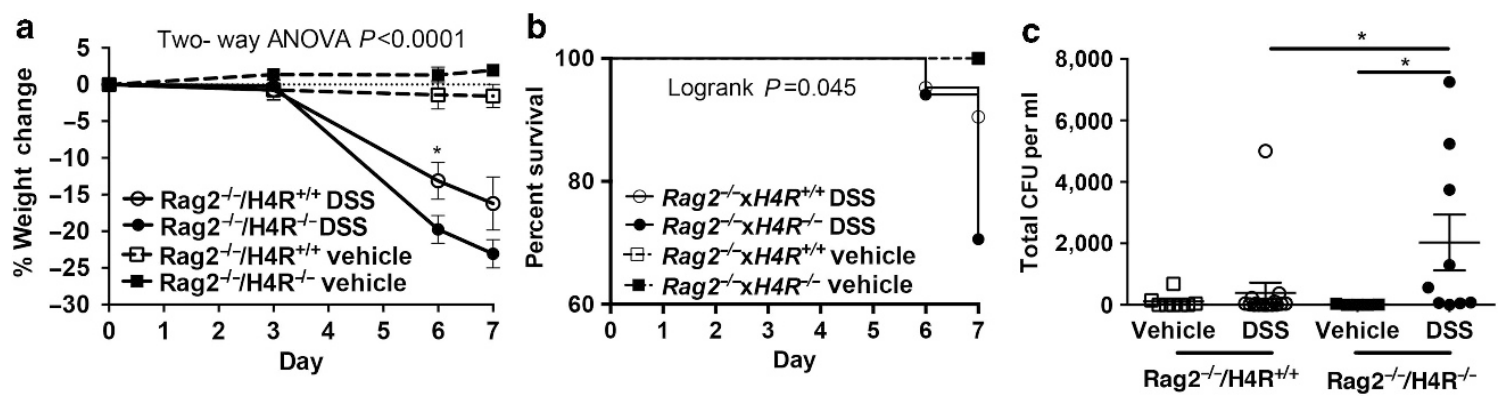

Figure 7 In the absence of functional adaptive immunity, histamine 4 receptor (H4R) protects against weight loss, death, and increased bacterial translocation to the mesenteric lymph node (MLN) after dextran sulfate sodium (DSS)-induced colitis. (a) Percentage weight change for DSS- or vehicle-treated Rag2 ${ }^{-/-}$and Rag2 ${ }^{-/-} \times \mathrm{H}_{4} \mathrm{R}^{-1-}$ mice $\left(n=5-17\right.$ mice/group). (b) Survival curve to day 7 in DSS- or vehicle-treated Rag2 $2^{-/-}$ and Rag2 ${ }^{-1-} \times \mathrm{H}_{4} \mathrm{R}^{-/-}$mice. (c) Colony-forming unit (CFU) counts from sterile MLN on blood agar plates on day 7 from DSS- or vehicle-treated Rag2 $^{-1-}$ and Rag2 $^{-1-} \times \mathrm{H}_{4} \mathrm{R}^{-1-}$ mice. ${ }^{*} P<0.05$. Data represented as mean \pm s.e.m. from three independent experiments.

effect of losing both adaptive immunity and this novel innate immune H4R-mediated pathway would be on mice undergoing experimental colitis. We thus crossed Rag $2^{-1-}$ mice (incapable of mounting adaptive immunity because of lacking $\mathrm{T}$ and $\mathrm{B}$ cells) to $H 4 R^{-{ }^{-}}$mice $\left(\mathrm{Rag}^{-{ }^{--}} \times H 4 \mathrm{R}^{-{ }^{-}}\right)$and performed DSS-induced colitis as Ox-induced colitis depends on T cells. We predicted that $\mathrm{Rag}^{-/-} \times H 4 \mathrm{R}^{-/-}$mice would show less disease severity than Rag2 ${ }^{-1-}$ mice, similar to their immunocompetent counterparts. To our surprise, $R a 2^{-1-} \times$ $H 4 R^{-\prime-}$ mice exhibited more profound weight loss and lower survival rates compared with $\mathrm{Rag} 2^{-/-}$mice (Figure 7a,b). $\mathrm{Rag}^{-{ }^{-1}} \times \mathrm{H}_{4} \mathrm{R}^{-1-}$ mice had significantly more bacteria disseminated into the regional mesenteric lymph nodes than Rag2 $2^{-/-}$mice (Figure 7c), indicating that septicemia was the probable cause of $\mathrm{Rag}^{-1-} \times \mathrm{H} 4 \mathrm{R}^{-1-}$ death. These results demonstrate that H4R-dependent innate inflammation is required to control bacterial invasion in the absence of adaptive immunity.

\section{DISCUSSION}

Although histamine is elevated in the mucosa of active UC, and urinary histamine metabolites correlate with disease activity, ${ }^{22,32}$ the mechanistic consequences of histamine release and receptor activation is lacking, limiting its consideration as a therapeutic target. Our work herein identifies a key role for MC-derived histamine release and subsequent $\mathrm{H} 4 \mathrm{R}$ activation in driving clinical and histopathologic severity in UC-like inflammation by regulating innate neutrophil recruitment and associated mucosal injury, likely via coordinating the downstream local production of IL-6 and neutrophil-attracting chemokines.

Although an early pilot study with ketotifen (an H1R blocker and MC stabilizer) showed some efficacy in a small subset of pediatric UC patients, ${ }^{33}$ the role of histamine receptors in the pathogenesis of colitis has remained unclear, with no clear role for $\mathrm{H} 1 \mathrm{R},{ }^{34,35}$ a proposed protective role for $\mathrm{H} 2 \mathrm{R}$ via microbial crosstalk, ${ }^{35,36}$ and preclinical evidence of a proinflammatory role for H4R. ${ }^{26,27,37}$ Our data demonstrate that mice lacking both H1R and H2R mount a competent response in experimental colitis and suggest that $\mathrm{MC}$-derived histamine functions via $\mathrm{H} 4 \mathrm{R}$ to exacerbate disease immunopathology. In patients, we noted a selective increase in $\mathrm{H} 4 \mathrm{R}$ over the other receptors; for H1R, this is in accordance with recent work, ${ }^{35}$ but $\mathrm{H} 2 \mathrm{R}$ upregulation previously described in a UC patient subset ${ }^{35}$ was not observed here. One explanation for this difference might be that the previous study compared inflamed with noninflamed tissue derived from the same individual, whereas we compared inflamed tissue from UC patients with control tissue from nonUC patients.

Our work focuses on determining how the histamine/HR pathway drives clinical and pathologic severity through innate and/or adaptive immune mechanisms. In addition to elevated $\mathrm{H} 4 \mathrm{R}$ transcripts in UC, we note that elevated H4R protein is found in proximity to areas of active MPO-associated cryptitis, a hallmark of UC. ${ }^{38}$ Building on previous findings, ${ }^{26,27,37}$ our data support the conclusion that H4R exacerbates immunopathology in experimental colitis, but the connections to innate inflammation presents a novel H4R-regulated pathway. Despite not placing mice on a histamine-free diet, our work defines a key role for endogenous MC-derived histamine in driving phenotypic severity. Although histamine levels are difficult to measure because of a lack of molecular stability in vivo, results from BMMC-reconstituted Sash mice define a signaling cascade whereby MC-derived histamine activates H4R to support neutrophil infiltration into the mucosa, likely through upstream regulation of neutrophil-attracting cytokine/ chemokine production. Precisely how H4R and the release of these mediators are connected--either directly or indirectly-as well as the identity of the H4R-bearing cell (discussed further below) remains to be determined. Although H4R may also function to limit the resolution of inflammation, the phenotype in $H 4 R^{-/-}$mice occurs during the inflammatory phase of the colitis models wherein cell influx into the tissue is a critical factor. Thus, colonic histamine/H4R helps to create a neutrophil-attracting milieu that promotes neutrophil migration into the mucosa, where they are poised to damage the crypts and drive clinical severity; importantly, our data do not rule out other H4R-mediated pathways to neutrophil infiltration, such as an indirect effect on intestinal barrier function, but these mechanisms remain to be determined in future studies. 
Overall, these observations reflect what is known to occur in IBD: the mucosa abnormally responds to bacterial signals, directing neutrophils to invade the mucosa and cause inadvertent damage to healthy tissue, a process sustained by signals like IL-6, IL-1 $\beta$, and tumor necrosis factor. ${ }^{10}$ Thus, this work is an important step forward, establishing that MCs, histamine, and H4R coordinate innate responses to drive clinical severity in IBD.

As the loss of H4R led to partial, but not complete, restoration of WT findings compared with vehicle controls, the sufficient role of non-H4R-dependent inflammation via adaptive immune pathways remains clear. Potentially, H4R signaling could provide an alternative target for IBD treatment differing from most current IBD therapies that target adaptive immune pathways; indeed, targeting innate immunity via $\mathrm{H} 4 \mathrm{R}$ may be a novel treatment consideration for UC patients with a limited response to $\mathrm{T}$-cell or other adaptive immune therapies (i.e., glucocorticoids).

In the context of $\mathrm{H} 4 \mathrm{R}$ promoting neutrophil-associated immunopathology, our work describes the following mechanistic scenarios: (i) activated local MCs produce histamine, (ii) histamine activates H4R-expressing cells, (iii) H4R signals either directly or indirectly to induce chronic neutrophilattracting chemokine release, and (iv) neutrophils invade the mucosa, simultaneously causing inadvertent damage to healthy tissue and protecting against sepsis by limiting bacteria that invade the damaged tissue. Thus, neutrophils in IBD likely play dual roles as (i) damage-inducing yet (ii) infection-fighting innate granulocytes. Indeed, our Rag2 ${ }^{-/-}$experiments highlight an important role for $\mathrm{H} 4 \mathrm{R}$ in protecting against bacterial invasion after mucosal barrier damage, and WT mice with experimental colitis are ultimately protected from lethal sepsis because of a compensatory balance between both innate and adaptive immunity. How can we disrupt the persistent vicious cycle of neutrophil-induced damage leading to bacterial translocation leading to more inflammation and more neutrophil recruitment? Perhaps blocking H4R may limit neutrophil invasion and "put the brakes" on perpetuating this cycle with a shut-off signal at the innate immune level, and this may be most successful in a specific set of patients that retain a fully functioning adaptive immune system.

Although not a pure Th2 disease, UC has clear evidence of Th2 activation (e.g., IL-13). ${ }^{39}$ The weight loss and pathologic inflammation still observed even after removing HDC or H4R signals suggested that histamine- and H4R-independent pathways led to inflammation persistence, likely via the well-described T-cell response in IBD. Supporting this conclusion, mucosal $\mathrm{CD} 4{ }^{+} \mathrm{T}$ cells and Th-associated cytokine (IL-4, IL-13, IL-17) transcript levels remained intact in $H D C^{-/-}$ and $H 4 R^{-1-}$ mice after experimental colitis induction. Thus, in contrast to the described function of H4R to skew $\mathrm{T}$ cells in Th2-mediated allergic asthma, ${ }^{40}$ histamine and H4R likely do not modulate adaptive mucosal immunity in experimental colitis models.

Interestingly, Mudter et al. ${ }^{41}$ described that the pleiotropic cytokine IL-6 limited T-cell apoptosis and enhanced T-cell survival in Ox-induced colitis; here, however, $H 4 R^{-1-}$ mice had diminished IL-6 compared with WT during experimental colitis but exhibited equal $\mathrm{CD} 4{ }^{+} \mathrm{T}$-cell numbers and $\mathrm{T}$ cellderived cytokines in the mucosa. Thus, we propose that colonic IL-6 released downstream of H4R likely has an innate function in colitis to help recruit neutrophils via a direct or indirect mechanism. H4R can support IL-6 production in an experimental peritonitis inflammation model, as $\mathrm{H} 4 \mathrm{R}$ blockade diminishes IL-6 production and the downstream neutrophil chemokines, CXCL1 and CXCL2. ${ }^{42}$ Moreover, IL-6 has been linked to neutrophil chemokine induction in colitis, as mice lacking gp130--an IL-6 receptor component--have reduced neutrophil chemokines after DSS colitis. ${ }^{43}$ We are actively pursuing the outstanding question of whether and which H4Rbearing cell produces IL-6 directly upon activation or through an indirect mechanism. A number of potential H4R-bearing candidate cells in the colon may participate in this process, some of which are known direct sources of IL-6 in the colon (innate cells such as MCs, neutrophils, and macrophages) and others that may play an intermediate role, like natural killer T cells. ${ }^{44}$ Notably, H4R blockers reduce IL- 6 production in several cell types including MCs, ${ }^{45-47}$ suggesting a common pathway between MC activation and production of this important cytokine; indeed, autocrine regulation of IL-6 by histamine-producing MCs themselves might be possible, as H4R agonists can stimulate IL-6 and neutrophilic chemokines from human MCs in vitro. ${ }^{48}$

Collectively, these data suggest that MC-derived histamine and H4R help to create an environment conducive to pathogenic neutrophil infiltration into the colonic mucosa, exacerbating the symptoms of experimental colitis. Alongside prior preclinical work utilizing H4R blockers in UC models, ${ }^{26,27,37}$ our work in human UC samples and experimental mouse models lends promise towards considering $\mathrm{H} 4 \mathrm{R}$ blockers as a therapeutic option for UC in patients who are not severely immunocompromised as well as a useful adjunct therapy to be used alongside current therapies targeting the adaptive immune system. However, more studies are needed to determine the safety of H4R blockers as induction agents or in the setting of coexisting infections based on the importance of the histamine-H4R-neutrophil innate immune axis demonstrated in our study.

\section{METHODS}

See Supplementary Methods for the following additional methods sections: Reagents, Monitoring of Disease Activity, Histology, Mast Cell Reconstitution, Real-Time PCR, and Colonic Cytokine Measurement.

Patient samples. Previously banked, institutional review boardapproved complementary DNA samples and unstained formalinfixed, paraffin embedded slides were obtained from Dr. Terrence Barrett (Northwestern University, Chicago, IL). Samples were collected from healthy, consented adults (age 18-70 years) undergoing surveillance colonoscopy for cancer found to have benign polyps (12 non-UC control patients; mean age $44 \pm 12$ years; $33 \%$ male) or individuals experiencing UC symptoms undergoing diagnostic testing, not currently on UC medications (14 UC patients; mean age $44 \pm 13$ 
years; 57\% male). Investigators were blinded to clinical information, including degree of disease severity for the UC patients.

Animals. Male and female (6-8 weeks old) C57BL/6J and Kit ${ }^{W-s h / W-s h}$ (Sash) mice (C57BL/6 background) were from the Jackson Laboratory (Bar Harbor, ME). $H^{-C^{-}}$mice (C57BL/6 background) have been previously described. ${ }^{49} H 4 R^{-1-}$ mice (BALB/c background) have been previously described. ${ }^{50} \mathrm{BALB} / \mathrm{c}$ and Rag $2^{-1-}$ mice (BALB/c background) were from Taconic (Hudson, NY). Rag2 ${ }^{-1-}$ mice were bred with $H 4 R^{-1-}$ mice to generate Rag $2^{-1-} \times H 4 R^{-1-}$ mice; Rag2 phenotype was assessed by flow cytometry for the absence of $\mathrm{CD} 3^{+}$ cells, and $H 4 R$ genotype was assessed by genotyping. $H 1 R^{-/-} \times$ $H 2 R^{-\prime-}$ double-knockout mice (C57BL/6 background) were obtained from Dr. Takeshi Watanabe (RIKEN Institute, Yokohama, Japan). All animal studies were performed under the guidelines for care and welfare by Institutional Animal Care and Use Committee under protocols approved by the Northwestern University Animal Care and Use Committee. All procedures were performed during the light cycle. Mice were housed in micro-isolator barrier cages (Lab Products, Seaford, DE) lined with coarse-grade Aspen Sani-Chips (Envigo, Indianapolis, IN) and provided with environmental enrichment including a Shepherd shack, Nestlet, Igloo, Safe Harbor retreat, and Nyla bones on a rotating schedule; high efficiency particulate airfiltered air circulated in the cages with positive air flow. Experimental mice were fed irradiated standard mouse diet (Envigo 7912): 5.8\% fat, $19.1 \%$ crude protein, and $44.3 \%$ carbohydrate. Breeder mice were fed irradiated breeder diet (Envigo 7912): 11.4\% fat, 17.2\% crude protein, and $45.2 \%$ carbohydrate. In an attempt to standardize the microbiome among all experimental mice, mice originally obtained from commercial vendors or other facilities were bred in-house for more than 3 generations to acclimate to the same environment before performing experiments.

Ox-induced colitis. Males (8-10 weeks old) were used for Ox-induced colitis in all experiments using $\mathrm{C} 57 \mathrm{BL} / 6, \mathrm{HDC}^{-/-}, \mathrm{BALB} / \mathrm{c}$, and $H 4 R^{-1-}$ mice. For experiments with Sash mice, mice were $11-12$ weeks old to allow for MC reconstitution in the gut. Mice were anesthetized, the abdomen was shaved $(2 \mathrm{~cm} \times 2 \mathrm{~cm}$ area), and $3 \%$ Ox in $100 \% \mathrm{EtOH}$ was applied and allowed to dry. After 1e week, anesthetized mice received 1\% Ox in EtOH/normal saline (1:1) enema, which was given $4 \mathrm{~cm}$ from the anal verge with a neonatal umbilical venous catheter (Utah Medical Products, Midvale, UT). Animal was held in the head-down position for $60 \mathrm{~s}$ after enema administration.

DSS-induced colitis. DSS colitis was given to 8-10-week-old female $\mathrm{BALB} / \mathrm{c}, \mathrm{H} 4 \mathrm{R}^{-/-}, \mathrm{Rag}^{-{ }^{-}}$, and $\mathrm{Rag}^{-{ }^{-}} \times \mathrm{H} 4 \mathrm{R}^{-I^{--}}$mice by administering 3.5\% DSS in water ad libitum for 6 days, followed by normal water until killing (water bottle changed every 3 days).

H4R immunohistochemistry. Formalin-fixed, paraffin-embedded slides were stained for H4R (ab188978, Abcam, Cambridge, MA). Relative H4R protein intensity was quantified using the Tissue Gnostics System (Vienna, Austria).

Myeloperoxidase stain and quantification. Anti-MPO staining was performed on formalin-fixed colonic tissue sections ( $4 \mu \mathrm{m})$. Lamina propria and intraepithelial MPO staining was quantified for each $40 \times$ high-powered field to determine average density of MPO-positive cells per high-powered field per section.

Assessment of bacterial translocation. Mesenteric lymph nodes were dissected under sterile conditions 7 days after initiating DSS colitis. Lysates were prepared in sterile phosphate-buffered saline, and $100 \mu \mathrm{l}$ was sterilely cultured on blood agar plates and incubated at $37^{\circ} \mathrm{C}$. Bacterial colonies were counted $96 \mathrm{~h}$ later.

Statistical analysis. Data are represented as the mean \pm s.e.m. unless otherwise noted. Statistical significance was determined using twotailed Student's $t$-test for two-group comparisons or analysis of variance (Dunnett's test) for more than two group differences. Two-way analysis of variance (Bonferroni's) was used to compare weight change between multiple groups over a time course. Analyses were performed using GraphPad Prism 5.0 (La Jolla, CA).

SUPPLEMENTARY MATERIAL is linked to the online version of the paper at http://www.nature.com/mi

\section{ACKNOWLEDGMENTS}

We thank Rob Thurmond and Paul Dunford at Johnson \& Johnson for providing the $H 4 R^{-1-}$ mice. We also thank Takeshi Watanabe (RIKEN Institute, Yokohama, Japan) for providing the $\mathrm{H} \mathrm{R}^{-1-} \times \mathrm{H} 2 \mathrm{R}^{-1-}$ doubleknockout mice. This project was financially supported in part by the National Institutes of Health (NIH) grant R01Al076456 (to P.J.B), NIH grant K08DK097721 (to J.B.W.), Northwestern University Allergy Immunology Research (NUAIR) T32 (5T32AI083216-03), and internal funding from Ann and Robert H. Lurie Children's Hospital of Chicago.

\section{AUTHOR CONTRIBUTIONS}

J.B.W., B.K.W., and P.J.B. contributed to the overall study concept and design; J.B.W., A.S., C-L.H., R.K-B., H.S., M.Y.W., R.C., L.M.A., J.B.B., B.K.W., and T.V. acquired the data; J.B.W., T.A.B., and P.J.B. analyzed and interpreted the data; J.B.W., M.L.M., and P.J.B. drafted the manuscript; P.J.B. critically revised the manuscript for important intellectual content; P.J.B., J.B.W., and B.K.W. obtained funding; T.A.B. provided human material support; P.J.B. supervised the study.

\section{DISCLOSURE}

The authors declared no conflict of interest.

c 2018 Society for Mucosal Immunology

\section{REFERENCES}

1. Fell, J.M., Muhammed, R., Spray, C., Crook, K. \& Russell, R.K. BSPGHAN IBD working group. Management of ulcerative colitis. Arch. Dis. Child. 101, 469-474 (2016).

2. de Souza, H.S. \& Fiocchi, C. Immunopathogenesis of IBD: current state of the art. Nat. Rev. Gastroenterol. Hepatol. 13, 13-27 (2016).

3. Fumery, M., Duricova, D., Gower-Rousseau, C., Annese, V., PeyrinBiroulet, L. \& Lakatos, P.L. Review article: the natural history of paediatriconset ulcerative colitis in population-based studies. Aliment. Pharmacol. Ther. 43, 346-355 (2016).

4. Romano, C., Syed, S., Valenti, S. \& Kugathasan, S. Management of acute severe colitis in children with ulcerative colitis in the biologics era. Pediatrics 137, pii: e20151184 (2016).

5. Brazil, J.C., Louis, N.A. \& Parkos, C.A. The role of polymorphonuclear leukocyte trafficking in the perpetuation of inflammation during inflammatory bowel disease. Inflamm. Bowel Dis. 19, 1556-1565 (2013).

6. Greenlee-Wacker, M.C. Clearance of apoptotic neutrophils and resolution of inflammation. Immunol. Rev. 273, 357-370 (2016).

7. Fournier, B.M. \& Parkos, C.A. The role of neutrophils during intestinal inflammation. Mucosal Immunol. 5, 354-366 (2012).

8. Masoodi, I. et al. Fecal lactoferrin, myeloperoxidase and serum C-reactive are effective biomarkers in the assessment of disease activity and severity in patients with idiopathic ulcerative colitis. J. Gastroenterol. Hepatol. 24, 1768-1774 (2009).

9. Masoodi, I. et al. Evaluation of fecal myeloperoxidase as a biomarker of disease activity and severity in ulcerative colitis. Dig. Dis. Sci. 57, 13361340 (2012).

10. Fonseca-Camarillo, G. \& Yamamoto-Furusho, J.K. Immunoregulatory pathways involved in inflammatory bowel disease. Inflamm. Bowel Dis. 21, 2188-2193 (2015).

11. Yadav, V., Varum, F., Bravo, R., Furrer, E., Bojic, D. \& Basit, A.W. Inflammatory bowel disease: exploring gut pathophysiology for novel therapeutic targets. Transl. Res. 176, 38-68 (2016).

12. Reuter, S., Stassen, M. \& Taube, C. Mast cells in allergic asthma and beyond. Yonsei Med. J. 51, 797-807 (2010). 
13. Fox, C.C., Lichtenstein, L.M. \& Roche, J.K. Intestinal mast cell responses in idiopathic inflammatory bowel disease. Histamine release from human intestinal mast cells in response to gut epithelial proteins. Dig. Dis. Sci. 38, 1105-1112 (1993)

14. Sander, L.E. et al. Selective expression of histamine receptors $\mathrm{H} 1 \mathrm{R}, \mathrm{H} 2 \mathrm{R}$, and H4R, but not H3R, in the human intestinal tract. Gut 55, 498-504 (2006).

15. Kotlyar, D.S., Shum, M., Hsieh, J., Blonski, W. \& Greenwald, D.A. Nonpulmonary allergic diseases and inflammatory bowel disease: a qualitative review. World J. Gastroenterol. 20, 11023-11032 (2014).

16. Sasaki, Y., Tanaka, M. \& Kudo, H. Differentiation between ulcerative colitis and Crohn's disease by a quantitative immunohistochemical evaluation of T lymphocytes, neutrophils, histiocytes and mast cells. Pathol. Int. 52, 277-285 (2002).

17. Yamagata, K., Tanaka, M. \& Kudo, H. A quantitative immunohistochemical evaluation of inflammatory cells at the affected and unaffected sites of inflammatory bowel disease. J. Gastroenterol. Hepatol. 13, 801-808 (1998).

18. King, T., Biddle, W., Bhatia, P., Moore, J. \& Miner, P.B. Jr. Colonic mucosal mast cell distribution at line of demarcation of active ulcerative colitis. Dig. Dis. Sci. 37, 490-495 (1992).

19. Boeckxstaens, G. Mast cells and inflammatory bowel disease. Curr. Opin. Pharmacol. 25, 45-49 (2015).

20. Nolte, H., Spjeldnaes, N., Kruse, A. \& Windelborg, B. Histamine release from gut mast cells from patients with inflammatory bowel diseases. Gut 31, 791-794 (1990).

21. Fox, C.C., Lazenby, A.J., Moore, W.C., Yardley, J.H., Bayless, T.M. \& Lichtenstein, L.M. Enhancement of human intestinal mast cell mediator release in active ulcerative colitis. Gastroenterology 99, 119-124 (1990).

22. Winterkamp, S. et al. Urinary excretion of N-methylhistamine as a marker of disease activity in inflammatory bowel disease. Am. J. Gastroenterol. 97, 3071-3077 (2002).

23. Bene, L. et al. Partial protection against dextran sodium sulphate induced colitis in histamine-deficient, histidine decarboxylase knockout mice. J. Pediatr. Gastroenterol. Nutr. 39, 171-176 (2004).

24. Garcia-Martin, E. et al. Severity of ulcerative colitis is associated with a polymorphism at diamine oxidase gene but not at histamine $\mathrm{N}$-methyltransferase gene. World J. Gastroenterol. 12, 615-620 (2006).

25. Neumann, D. \& Seifert, R. The therapeutic potential of histamine receptor ligands in inflammatory bowel disease. Biochem. Pharmacol. 91, 12-17 (2014).

26. Varga, C., Horvath, K., Berko, A., Thurmond, R.L., Dunford, P.J. \& Whittle, B.J. Inhibitory effects of histamine $\mathrm{H} 4$ receptor antagonists on experimental colitis in the rat. Eur. J. Pharmacol. 522, 130-138 (2005).

27. Schirmer, B., Rezniczek, T., Seifert, R. \& Neumann, D. Proinflammatory role of the histamine $\mathrm{H} 4$ receptor in dextrane sodium sulfate-induced acute colitis. Biochem. Pharmacol. 98, 102-109 (2015).

28. Smolinska, S., Jutel, M., Crameri, R. \& O'Mahony, L. Histamine and gut mucosal immune regulation. Allergy 69, 273-281 (2014).

29. Rogler, G. \& Andus, T. Cytokines in inflammatory bowel disease. World J. Surg. 22, 382-389 (1998).

30. Weigmann, B. \& Neurath, M.F. Oxazolone-induced colitis as a model of Th2 immune responses in the intestinal mucosa. Methods Mol. Biol. 1422, 253-261 (2016).

31. Catana, C.S., Berindan Neagoe, I., Cozma, V., Magdas, C., Tabaran, F. \& Dumitrascu, D.L. Contribution of the IL-17//L-23 axis to the pathogenesis of inflammatory bowel disease. World J. Gastroenterol. 21, 5823-5830 (2015).

32. Raithel, M., Matek, M., Baenkler, H.W., Jorde, W. \& Hahn, E.G. Mucosal histamine content and histamine secretion in Crohn's disease, ulcerative colitis and allergic enteropathy. Int. Arch. Allergy Immunol. 108, 127-133 (1995).
33. Jones, N.L., Roifman, C.M., Griffiths, A.M. \& Sherman, P. Ketotifen therapy for acute ulcerative colitis in children: a pilot study. Dig. Dis. Sci. 43, 609615 (1998).

34. Raithel, M. et al. Plasma histamine levels $(H)$ during adjunctive $\mathrm{H} 1$-receptor antagonist treatment with loratadine in patients with active inflammatory bowel disease (IBD). Inflamm. Res. 59 ((Suppl 2)), S257S258 (2010).

35. Smolinska, S. et al. Histamine receptor 2 is required to suppress innate immune responses to bacterial ligands in patients with inflammatory bowel disease. Inflamm. Bowel Dis. 22, 1575-1586 (2016).

36. Gao, C. et al. Histamine $\mathrm{H} 2$ receptor-mediated suppression of intestinal inflammation by probiotic Lactobacillus reuteri. MBio 6, e01358-15 (2015).

37. Fogel, W.A., Jochem, J. \& Lewinski, A. Influence of the H3/H4 receptor antagonist, thioperamide on regional haemodynamics in rats with trinitrobenzene sulfonic acid-induced colitis. Inflamm. Res. 56 ((Suppl 1)), S21-S22 (2007).

38. Masoodi, I., Tijjani, B.M., Wani, H., Hassan, N.S., Khan, A.B. \& Hussain, S. Biomarkers in the management of ulcerative colitis: a brief review. Ger. Med. Sci. 9, Doc03 (2011).

39. Li, J. et al. Profiles of lamina propria T helper cell subsets discriminate between ulcerative colitis and Crohn's disease. Inflamm. Bowel. Dis. 22, 1779-1792 (2016).

40. Thurmond, R.L., Gelfand, E.W. \& Dunford, P.J. The role of histamine H1 and $\mathrm{H} 4$ receptors in allergic inflammation: the search for new antihistamines. Nat. Rev. 7, 41-53 (2008).

41. Mudter, J. et al. The transcription factor IFN regulatory factor-4 controls experimental colitis in mice via T cell-derived IL-6. J. Clin. Invest. 118, 2415-2426 (2008).

42. Strakhova, M.I. et al. In vitro and in vivo characterization of A-940894: a potent histamine $\mathrm{H} 4$ receptor antagonist with anti-inflammatory properties. Br. J. Pharmacol. 157, 44-54 (2009).

43. Matsumoto, S. et al. Essential roles of IL-6 trans-signaling in colonic epithelial cells, induced by the IL-6/soluble-IL-6 receptor derived from lamina propria macrophages, on the development of colitis-associated premalignant cancer in a murine model. J. Immunol. 184, 1543-1551 (2010).

44. Leite-de-Moraes, M.C. et al. Cutting edge: histamine receptor $\mathrm{H} 4$ activation positively regulates in vivo IL-4 and IFN-gamma production by invariant NKT cells. J. Immunol. 182, 1233-1236 (2009).

45. Simon, T., Laszlo, V., Lang, O., Buzas, E. \& Falus, A. Histamine regulates relevant murine dendritic cell functions via $\mathrm{H} 4$ receptor. Front. Biosci. (Elite Ed.) 3, 1414-1424 (2011).

46. Dunford, P.J., O'Donnell, N., Riley, J.P., Williams, K.N., Karlsson, L. \& Thurmond, R.L. The histamine $\mathrm{H} 4$ receptor mediates allergic airway inflammation by regulating the activation of CD4 + Tcells. J. Immunol. 176, 7062-7070 (2006).

47. Desai, P. \& Thurmond, R.L. Histamine H(4) receptor activation enhances LPS-induced IL-6 production in mast cells via ERK and PI3K activation. Eur. J. Immunol. 41, 1764-1773 (2011).

48. Jemima, E.A., Prema, A. \& Thangam, E.B. Functional characterization of histamine $\mathrm{H} 4$ receptor on human mast cells. Mol. Immunol. 62, 19-28 (2014).

49. Rahman, M.A., Inoue, T., Ishikawa, T., Yatsuzuka, R., Ohtsu, H. \& Kamei, C. Involvement of chemical mediators in nasal allergic responses of HDC-KO mice. Eur. J. Pharmacol. 567, 245-251 (2007).

50. Hofstra, C.L., Desai, P.J., Thurmond, R.L. \& Fung-Leung, W.P. Histamine $\mathrm{H} 4$ receptor mediates chemotaxis and calcium mobilization of mast cells. J. Pharmacol. Exp. Ther. 305, 1212-1221 (2003). 